\title{
Flow imaging in vivo using off resonance spin labeling induced by extraneous contrast agent
}

\author{
Jessica A Bastiaansen ${ }^{1 *}$, Jean-Baptiste Ledoux ${ }^{1,2}$, Andrew J Coristine ${ }^{1}$, Helene Feliciano ${ }^{1}$, Debora Bonvin ${ }^{3}$, \\ Marijana Mionic ${ }^{3,1}$, Heinrich Hofmann ${ }^{3}$, Matthias Stuber ${ }^{1,2}$ \\ From 19th Annual SCMR Scientific Sessions \\ Los Angeles, CA, USA. 27-30 January 2016
}

\section{Background}

Tissues within the dipolar field of superparamagnetic contrast agents experience a frequency shift that enables positive contrast MRI with acquisition schemes using on-resonant saturation (Figure 1a,b). The administration of iron oxide based agents enables a plethora of applications using positive contrast MRI. They have been widely explored and mainly focused on lymphography, angiography, cancer detection and atherosclerosis. In this preliminary in vivo study, a dynamic contrast mechanism is presented,using externally placed suspensions of iron oxide nanoparticles (SPIONs) to induce noninvasive spin tagging of nearby blood flow.

\section{Methods}

A cylindrical tube containing a concentrated suspension of $19 \mathrm{~nm}$ SPIONs was placed alongside the upper leg in human volunteers (Figure 1c), causing a local magnetic field change in the adjacent tissue. Protons residing in the off-resonance field experience a frequency shift as they move through or are static within this field. A 3D gradient echo based MRI acquisition, preceded by a narrow or broad bandwidth $(100 \mathrm{~Hz}$ or $2 \mathrm{kHz})$ on-resonant saturation pulse (Figure 1d), was used for data acquisition on a 3T clinical MRI scanner. This led to a selective excitation of the off resonance area (Figure 1c). A variable radio frequency excitation angle and delay time ranging from 0 to $0.8 \mathrm{~s}$ facilitated the tagging of the off-resonant flowing spins in the nearby veins and arteries. Flowing spins remain unaffected by the narrowband RF pulse and are tagged by the external SPIONs.

\section{Results}

The off-resonance area was selectively excited and visualized with a $0 \mathrm{~s}$ delay time (Figure 1e). Spins were tagged up to $8 \mathrm{~cm}$ from the tissue surface with a $100 \mathrm{~Hz}$ suppression bandwidth. By extending the delay time between saturation pulse and acquisition, flowing protons that were within the SPIONs affected area still showed positive contrast in further downstream areas and their motion along the femoral vein and arteries could be tracked up to 6.7 $\mathrm{cm}$ showing a poiseuille shaped velocity profile (Figure $1 \mathrm{e}-\mathrm{h})$. A calculated blood velocity of $8.4 \mathrm{~cm} / \mathrm{s}$ was within range of published values of $13.9 \pm 5.9 \mathrm{~cm} / \mathrm{s}$. The surrounding tissue experienced magnetization recovery due to T1 and was excited with increasing RF excitation angles and delay time, in order to enhance tissue contrast. Applying a broadband RF pulse compensated for T1 recovery of surrounding tissue when subtracted from narrowband images (Figure 2). Fat saturation enhanced the positive contrast, although with unwanted saturation of a frequency band around $400 \mathrm{~Hz}$, which suppressed nearly half of the off-resonant area between $3.4 \mathrm{~cm}$ and $6.2 \mathrm{~cm}$ from the skin.

\section{Conclusions}

Flow imaging with iron induced spin tagging (FLIRT) was achieved using external SPIONs which targeted the positive labeling of moving protons. The feasibility of this method was shown in vivo with preliminary data obtained in human volunteers. It allows for flow visualization in the vicinity of ferrimagnetic objects without contrast injection. 


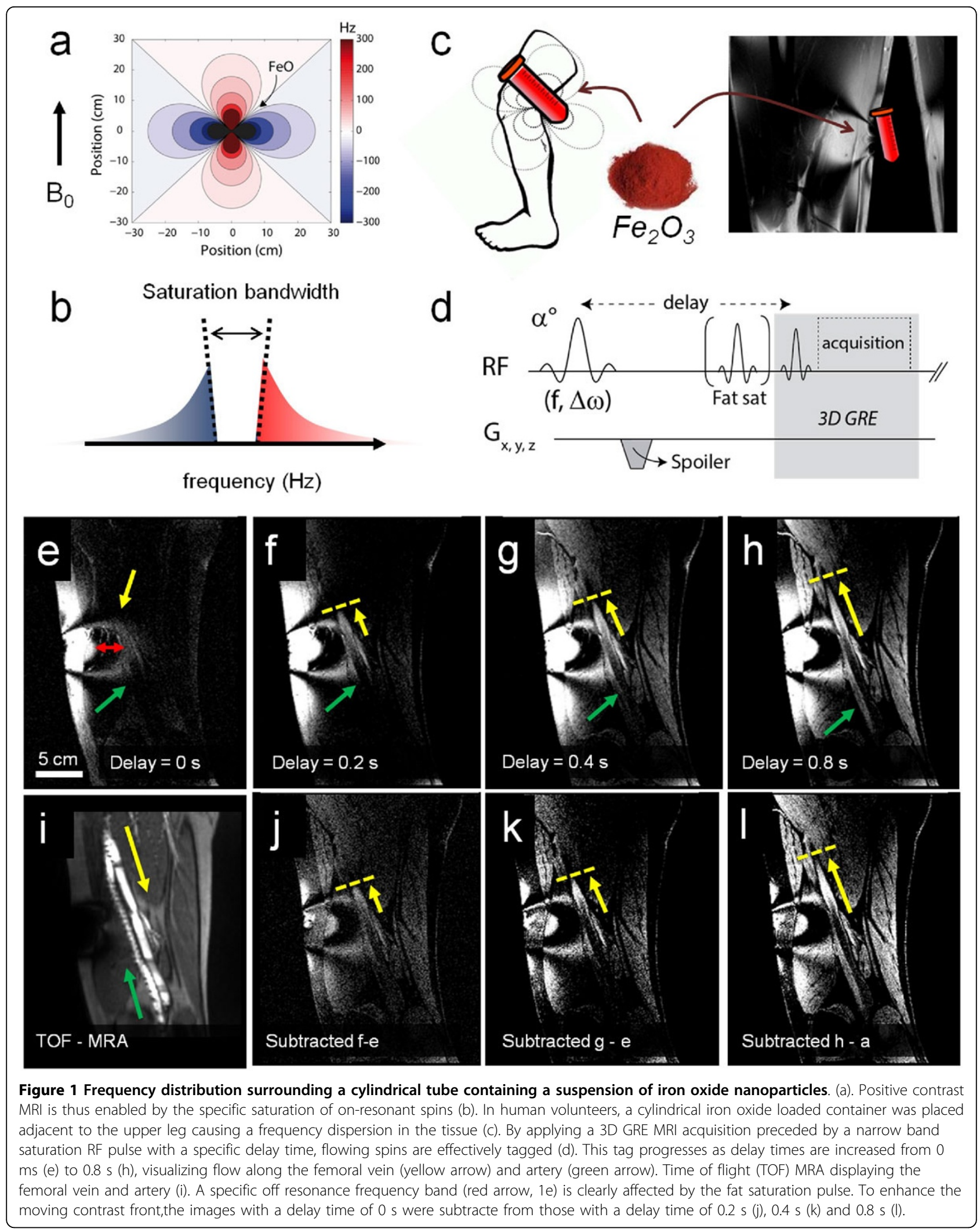




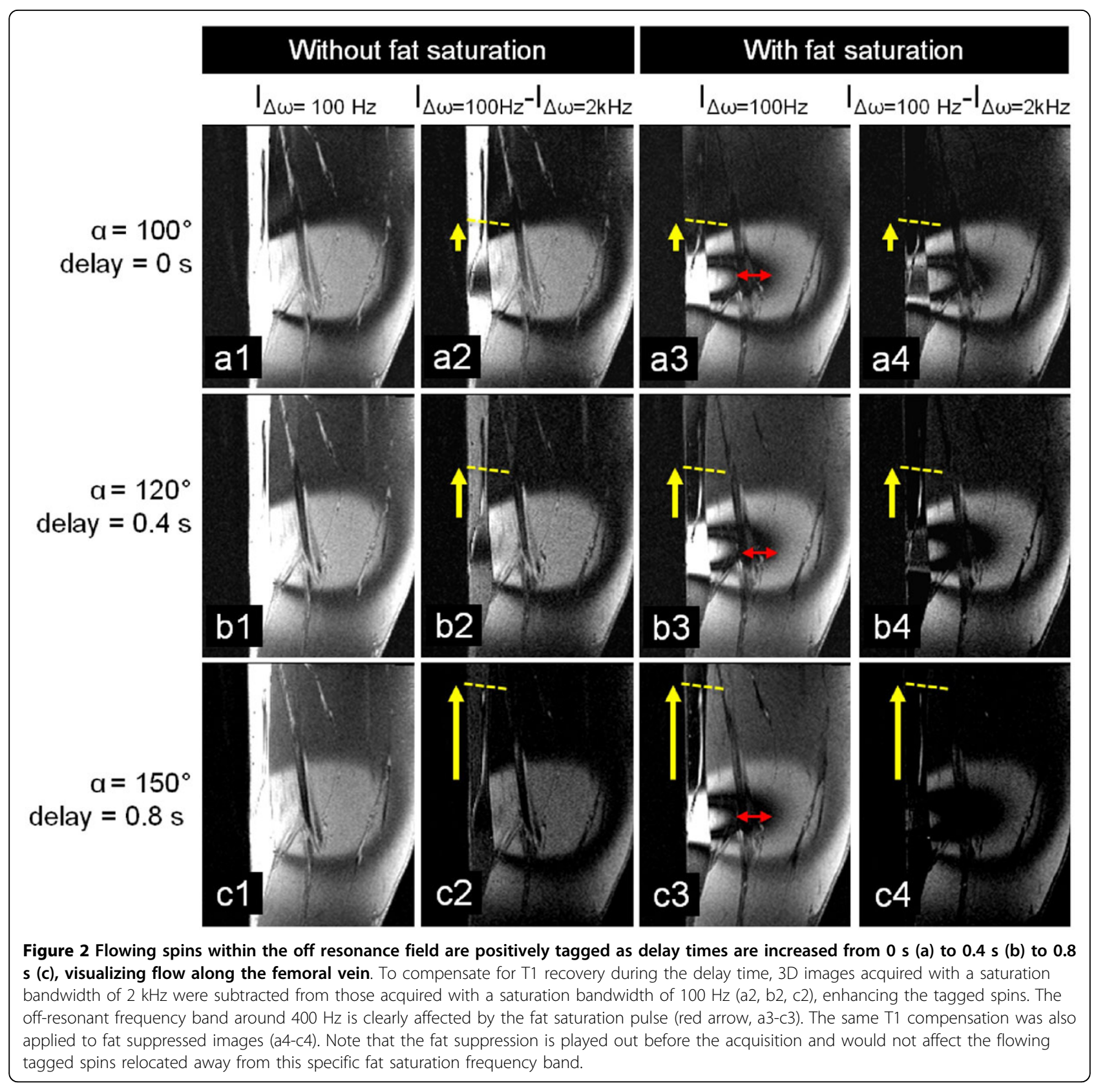

Published: 27 January 2016

doi:10.1186/1532-429X-18-S1-P258

Cite this article as: Bastiaansen et al:. Flow imaging in vivo using off

resonance spin labeling induced by extraneous contrast agent. Journal

of Cardiovascular Magnetic Resonance 2016 18(Suppl 1):P258. 